\title{
Deposition and Growth of Functional Nanomaterials by LDW and MAPLE Techniques
}

\author{
Angel Perez del Pino \\ Institute of Materials Science of Barcelona, ICMAB-CSIC, Campus UAB, 08193 Cerdanyola del Valles, Spain; \\ aperez@icmab.es
}

Received: 10 November 2020; Accepted: 16 November 2020; Published: 23 November 2020

Actual societal challenges require a vigorous progress on functional materials with improved functionalities. Thus, a huge variety of organic, inorganic and hybrid materials are being developed for going beyond the state-of-the-art in key applications such as sensing, energy production and management, waste treatment, electronics, etc. Besides, innovative fabrication techniques are also emerging as solid alternatives to conventional methods with the aim of not only achieving the synthesis of advanced materials in a cost-effective and eco-friendly manner, but also with the best possible integration degree in devices.

Laser technologies stand out when applied to material synthesis and processing due to the fast and powerful nature of laser radiation, its ability to be focused in small areas, as well as the facile integration of these technologies in the industrial environment. Pulsed laser radiation can initiate a myriad of complex photochemical and photothermal mechanisms in the irradiated material, provoking fast heating, chemical reactions, recrystallization, surface melting, hydrodynamic processes, ablation, etc. Thereby, the direct surface transformation of the irradiated material can be attained in a fast and easy way, and with high spatial resolution through laser direct write (LDW) technologies. It is worth noticing that not only localized chemical transformations can be achieved through LDW, but also additive/subtractive manufacturing in the microscale, stablishing innovative paths to the fabrication of a plethora of integrated devices. Therefore, LDW comprehends a variety of approaches allowing for "printing" on surface materials without the use of molds or masks, so customizable processes can be easily done. A different philosophy is accomplished in laser-based deposition of materials, in which the objective is the highly controlled growth of functional thin films over larger areas. Pulsed laser deposition (PLD) is the paradigm of this family of techniques, in which the intense laser-induced ablation of solid targets in controlled gas environments allow for the growth of highly crystalline inorganic thin films even composed of complex materials. It is important to note that during PLD the irradiated material of the target is decomposed in highly energetic ionic species, which are transferred in the form of expanding plasma towards a facing substrate. The arriving species diffuse at the surface and nucleate, allowing the growth of the film. Therefore, this method is not suitable to the direct deposition of organic molecules as polymers or nanostructures. Nevertheless, the matrix assisted pulsed laser evaporation (MAPLE) is a PLD-like method that allows the transfer of molecular species/nanostructures without damage. MAPLE uses a frozen target composed of a solution/dispersion of the entities of interest in which the solvent absorbs the laser radiation, protecting the entities, and experiences an explosive boiling leading to the transfer of the species towards the facing substrate. Thus, by means of PLD and MAPLE, the laser deposition of either organic, inorganic or hybrid advanced materials is possible.

This Special Issue of Crystals is focused on LDW and MAPLE techniques providing cutting-edge works that illustrate the versatility of such methods. First of all, Prof. Camacho-López et al. [1] present the spatially confined growth of molybdenum oxides through a LDW method by means of femtosecond laser irradiation. Molybdenum oxides show potential application to a rich variety of technologic 
fields as sensors, energy storage, photonics or catalysis. By focusing $60 \mathrm{fs}$ laser pulses, the authors irradiated about $100 \mu^{2}$ regions of crystalline Mo films in air with different irradiation times and laser fluence conditions, always below the ablation threshold of Mo. A complex coupling of optical and thermal mechanisms led to spatially resolved phase transitions, prompting the formation of various types of $\mathrm{MoO}_{\mathrm{x}}$ as well as different types of nano- and microstructures (nanostructured islands and nanobars, lamellar and polyhedral morphologies, etc.) The appearance and distribution of such phases and morphologies are highly correlated to the used integrated laser fluence opening an experimental window for the controlled growth of such functional oxides in an easy and versatile way.

As stated above, LDW comprehends different approaches for attaining the printing of functional materials. Among them, laser-induced forward transfer (LIFT) stands out as a method that allows the transfer of small volumes of material on receiving substrates by means of the spatially resolved laser irradiation of thin layer donors. An extensive amount of works has been reported about the transfer of solid and even paste materials. However, in this Special Issue, Fernández-Pradas et al. [2] review the use of the LIFT method for the transfer of functional inks. The coupling between optical and rheological properties of the ink donor film leads to the emergence of complex phenomena that can provoke the formation of a liquid jet, allowing the transfer of an ink droplet which, once dried, forms a micrometric pixel at the receiving substrate. The hydrodynamic processes that determine the final size and shape of the pixel also depend on the type of laser radiation (continuous wave or pulsed) and laser energy, besides the wetting properties of the ink on the receiving substrate. Printing of continuous tracks is also possible by laser scanning and relative movement between donor layer and receiving substrate, though additional challenges appear for obtaining straightforward overlapping of contiguous pixels. In summary, LIFT allows the controlled transfer of a wide range of functional inks containing biomolecules, polymers or metallic nanostructures, with high versatility and avoiding many restrictions of conventional printing methods.

Regarding the MAPLE deposition of functional thin films constituted by delicate materials, Ajnsztajn et al. [3] present an innovative work related to the deposition of transparent and supercapacitive MXene-polymer films by means of resonant infrared matrix-assisted pulsed laser evaporation (RIR-MAPLE). In this technique, partitioned frozen targets containing polyflourene (PFO) and $\mathrm{Ti}_{3} \mathrm{C}_{2} \mathrm{~T}_{\mathrm{x}}$ nanosheets were submitted to pulsed laser radiation with peak at $2.94 \mu \mathrm{m}$ wavelength with the aim to excite the hydroxyl bonds of water molecules and prompt the fast evaporation of the solvent. In this way, the water vapor gently transfers the PFO molecules and MXene nanosheets leading to homogeneous polymer/nanoparticle composite films. Highly transparent hybrid electrodes with capacitance in the range of tens of $\mathrm{mF} / \mathrm{cm}^{2}$ were obtained revealing the high versatility of this method for obtaining multi-functional films.

Finally, Queraltó et al. [4] present another work about MAPLE deposition of hybrid films, also using water-ice as matrix, but through a totally different approach. In this case, the frozen targets were composed of perovskite $\mathrm{BiFeO}_{3}$ and $\mathrm{LaFeO}_{3}$ nanofibers (obtained by electrospinning) and graphene oxide (GO) sheets. UV pulsed laser radiation, which is not absorbed by the water-ice matrix, is submitted to the frozen dispersion leading to the fast heating of the oxide nanofibers and GO sheets, provoking the vaporization of the surrounding matrix and the deposition of the nanoentities (inverse MAPLE). In this way, composite layers constituted by mixtures of nanofibers fragments and reduced GO sheets were obtained revealing high photocatalytic efficiency for the degradation of methyl orange dye, both under UV and visible light irradiation conditions.

To conclude, I believe that this Special Issue on Deposition and Growth of Functional Nanomaterials by LDW and MAPLE Techniques offers an inspiring view on the versatility and potential applications of these technologies. I wish to express my deepest gratitude to all authors who contributed with such excellent quality manuscripts to this Special Issue in such difficult pandemic times. I also want to thank the Editorial Office of Crystals for the professional management of the manuscripts carried out during the whole submission process and for the provided help.

Conflicts of Interest: The author declares no conflict of interest. 


\section{References}

1. Camacho-Lopez, S.; Cano-Lara, M.; Camacho-Lopez, M. Fast Growth of Multi-Phase MoO ${ }_{x}$ Synthesized by Laser Direct Writing Using Femtosecond Pulses. Crystals 2020, 10, 629. [CrossRef]

2. Fernández-Pradas, J.M.; Serra, P. Laser-Induced Forward Transfer: A Method for Printing Functional Inks. Crystals 2020, 10, 651. [CrossRef]

3. Ajnsztajn, A.; Ferguson, S.; Thostenson, J.O.; Ngaboyamahina, E.; Parker, C.B.; Glass, J.T.; Stiff-Roberts, A.D. Transparent MXene-Polymer Supercapacitive Film Deposited Using RIR-MAPLE. Crystals 2020, 10, 152. [CrossRef]

4. Queraltó, A.; György, E.; Ivan, R.; Pérez del Pino, Á.; Frohnhoven, R.; Mathur, S. Enhanced UV-Vis Photodegradation of Nanocomposite Reduced Graphene Oxide/Ferrite Nanofiber Films Prepared by Laser-Assisted Evaporation. Crystals 2020, 10, 271. [CrossRef]

Publisher's Note: MDPI stays neutral with regard to jurisdictional claims in published maps and institutional affiliations.

(C) 2020 by the author. Licensee MDPI, Basel, Switzerland. This article is an open access article distributed under the terms and conditions of the Creative Commons Attribution (CC BY) license (http://creativecommons.org/licenses/by/4.0/). 